\title{
Outage Analysis of Clustered Cooperative Networks in Generalized Fading and Shadowing
}

\author{
Nemanja Zdravković, Student Member, IEEE
}

\begin{abstract}
In this paper, the outage probability of a noiselimited decode-and-forward wireless cooperative network cluster, subject to the Nakagami-m multipath fading and Gamma shadowing is investigated. The closed-form expression for outage probability, when the network fusion center selects the stronger received transmission out of the maximal ratio combined signal, arriving from the nodes acting as relays, and a repeated signal from the originating node, is derived. Moreover, special cases, in which the generalized fading and shadowing channel is reduced to several familiar propagation environments, are observed. The derived analytical expressions for outage probability are furthermore confirmed by running independent MonteCarlo simulations. The effects of multipath fading severity and shadowing sharpness, average signal-to-noise ratio values, and network dimension on outage performance are discussed.
\end{abstract}

Keywords - Cooperative networks, decode-and-forward relay, multipath fading, outage probability, shadowing.

\section{INTRODUCTION}

$\mathrm{C}$ OOPERATIVE wireless communication systems acting as virtual antenna arrays provide full spatial diversity by exploiting numerous independent propagation paths within the wireless network, improving overall performance [1]. The application of cooperative strategies, both in cellular, and in ad/hoc and sensor networks has raised interest in future communication networks as we move towards 5G [2-4]. Protocols that are usually employed allow nodes equipped with a single antenna to cooperate by forwarding each other's data. These protocols are categorized into two major groups amplify-and-forward (AF) and decode-and-forward (DF). In the former group, a node simply amplifies its partners' data, relaying it to a fusion center with either a fixed or

Paper received May 2, 2016; revised September 28, 2016; accepted November 3, 2016. Date of publication November 20, 2016. The associate editor coordinating the review of this manuscript and approving it for publication was Prof. Grozdan Petrović.

This paper is a revised and expanded version of the paper presented at the 23rd Telecommunications Forum TELFOR 2015 [21].

This paper was supported by the Norwegian Ministry for Foreign Affairs under the project "Norwegian, Bosnian and Serbian cooperation platform for university and industry ICT R\&D" - NORBAS (grant 2011/1383) headed by NTNU.

Nemanja Zdravković is with the Faculty of Electrical Engineering, University of Niš, Aleksandra Medvedeva 14, 18000 Niš, Serbia and with the Department of Electronics and Telecommunications, Norwegian University of Science and Technology - NTNU, Høgskoleringen 1, 7491 Trondheim, Norway (phone: 381-64-2808100; e-mail: nemanja.zdravkovic@elfak.ni.ac.rs). variable gain, while in the latter group a node will first decode its partners' data, re-encode, and then forward. Often, AF protocols require channel state information between cooperating nodes, and this may not be the case in many real-world scenarios [5]. Both protocol groups achieve full spatial diversity, improving overall network performance.

Cooperation protocols however, have their fundamental limits, as pointed out in [6]. The benefits of cooperation can be exploited by finite-sized and partially connected network clusters, but large-scale networks cannot. Receivers can only focus on a small number of strong nearby transmitters, ignoring distant ones, leading to receiver near-sightedness and a clustered network structure. In other words, cooperation cannot change an interference-limited network to a noise-limited one [6]. Therefore, in this paper, the analysis presented is limited to a small-to-medium sized network, which can be considered clustered and hence noise-limited.

Performance analysis of DF cooperative wireless networks was performed in [2-12]. Outage probability over Rayleigh and Nakagami- $m$ fading channels was analyzed in [2] and [3], respectively. Outage performance and packet error rates in DF networks subject to Rayleigh fading employing repetition schemes to compensate for unsuccessfully decoded packets were analyzed in [7-9]. Exact and asymptotic outage behavior of low-latency cooperative networks set in a dynamic environment prone to link blockage, were examined in [10]. The presence of shadowing and its effects on overall network performance was analyzed in [11], and the combined effects of multipath fading and shadowing were addressed in [12].

Whereas in [12], outage performance was analyzed when the destination applied maximal ration combining (MRC) on the initial and relayed copies only, this paper is encouraged by the repetition protocols in [7]. Namely, in this paper, the cooperative network communicates in a manner where the fusion center aggregates all the data from all nodes using a two-level combining mechanism. The aim of this mechanism is to achieve a trade-off between power gain coming from the repetitions, and diversity gain coming from the cooperating nodes, obtaining optimal performance. To the best of the author's knowledge, the derived outage probability expressions for the generalized fading and shadowing, applying the proposed two-level combining mechanism are novel and can be used for designing a cooperative wireless network cluster in a wide range of propagation environments. 
The rest of the paper is organized as follows. Section II introduces the system and channel model. Novel expressions for outage probability are derived in Section III, and special cases are treated in Section IV. Section V presents the numerical results with appropriate discussions, and concluding remarks are given in Section VI.

\section{CHANNEL AND SYSTEM MODEL}

In this Section, the composite channel model and its parameters affecting the cooperative network are described. Afterwards, the system model and cooperation mechanism is presented.

\section{A. Channel model}

Nodes in the network communicate with the fusion center and cooperate with each other over a composite fading and shadowing channel. The multipath portion of the compound fading and shadowing channel is described by the Nakagami- $m$ distribution, which accounts for realworld fading scenarios. The network can be set in various line-of-sight (LoS) and non-line-of-sight (NLoS) environments, as well as severe fading, even severer than the well-known and often considered Rayleigh model. The shadowing effects depend on the local topology and receiver surroundings and characteristically appear in industrial wireless network environments [13]. The effects of shadowing are often modelled by the log-normal distribution; however, with appropriate parameter approximation, shadowing effects can be described by the Gamma distribution [14-16]. The composite NakagamiGamma fading/shadowing channel is often encountered in literature as the generalized- $K\left(K_{G}\right)$ channel [17]. The probability density function (PDF) of a $K_{G}$ fading envelope is given as [17]

$$
f(x)=\frac{4 m^{\frac{k+m}{2}} x^{k+m+1}}{\Gamma(m) \Gamma(k) \Omega^{\frac{k+m}{2}}} K_{k-m}\left(2 \sqrt{\frac{m}{\Omega}} x\right),
$$

where $k$ and $m$ are the shadowing and fading severity parameters, respectively, $\Omega$ is the mean power defined as $\Omega=\mathrm{E}\left[x^{2}\right] / k$, with $\mathrm{E}[\cdot]$ denoting the expectation operator. Furthermore, $\Gamma(\cdot)$ is the Gamma function defined in [18, eq. (1.1)] and $K_{1}(\cdot)$ is the $v$-th order modified Bessel function of the second kind, defined in [18, eq. (8.432)]. A variety of channel models can be obtained by setting certain values of the fading and shadowing severity parameters. For instance, setting $m=1$ accounts for the multipath portion of the channel being Rayleigh distributed, reducing the $K_{G}$ channel to the co-called $K$ channel. Moreover, letting $k \rightarrow \infty$ the effects of shadowing are diminished. Letting both $k \rightarrow \infty$ and $m \rightarrow \infty$, the compound fading channel is reduced to an additive white Gaussian noise (AWGN) channel. The shadowing severity parameter $k$ is related to the shadowing spread parameter in log-normal shadowing as $[15,16]$

$$
\sigma_{S H}[\mathrm{~dB}]=10 / \ln 10 \sqrt{\psi^{\prime}(k)},
$$

where $\psi^{\prime}$ is the first derivative of the digamma function, defined in $[8$, eq. (8.360)]. Denote the instantaneous received signal-to-noise ratio (SNR) per symbol with $\gamma=x^{2} \times E_{S} / N_{0}$, with $E_{S}$ denoting symbol energy and $N_{0}$ denoting the single-sided power spectral density of the noise. The average SNR per symbol is therefore $\bar{\gamma}=k \Omega E_{S} / N_{0}$. The PDF of the received SNR is obtained using a random variable (RV) transform as [17]

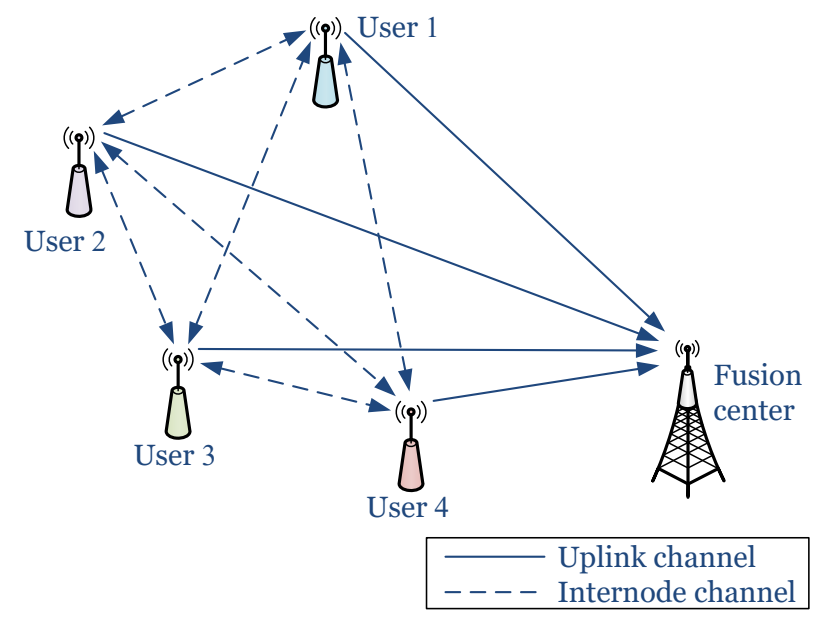

Fig. 1 System model of a cooperative network cluster consisting of $M=4$ nodes and the fusion center.

$$
f(\gamma)=\frac{2 \Xi^{\frac{k+m}{2}}}{\Gamma(m) \Gamma(k)} \gamma^{\frac{k+m}{2}-1} K_{k-m}(2 \sqrt{\Xi \gamma}),
$$

with $\Xi=k m / \bar{\gamma}$. The cumulative distribution function (CDF) of $\gamma$, can be obtained in closed-form as [17]

$$
F(\gamma)=\frac{1}{\Gamma(m) \Gamma(k)} G_{1,3}^{2,1}\left(\Xi \gamma \mid \begin{array}{ccc}
1 & \\
m, & k, & 0
\end{array}\right),
$$

where $G(\cdot)$ is the Meijer's $G$-function, defined in [18, eq. (9.301)]. It should be noted that the CDF is expressed in terms of the Meijer's $G$-function, which is built-in function in Mathematica software package. Furthermore, Meijer's $G$-functions can be transformed to more familiar hypergeometric functions [18, eq. (9.41.1)] by applying the relation [19, eq. (07.34.26.0004.01)]. Through the use of (4), outage probability is obtained replacing $\gamma$ with an outage threshold $\gamma$.

\section{B. System model}

The wireless cooperative network cluster under analysis consists of $M$ user nodes, denoted with $U_{i}, i=1, \ldots, M$, and the fusion center, as shown in Fig. 1. User nodes act as data sources and relays, while the fusion center acts as the destination. Cooperation between the nodes is performed in two stages. In the first stage, each node broadcasts its own data packet to the fusion center via the uplink channel, and to the other nodes via the internode channels in one time step, as shown in Fig. 2 (a) at the top of the next page. In the second stage of cooperation, each node will forward a packet that has been successfully decoded to the fusion center. For those packets a node has failed to decode, it will re-send its own data packets. Node $U_{i}$ "knows" the number of nodes that have successfully decoded its packets via feedback messages, which are assumed to be error-free. Node $U_{i}$ only re-sends only those 


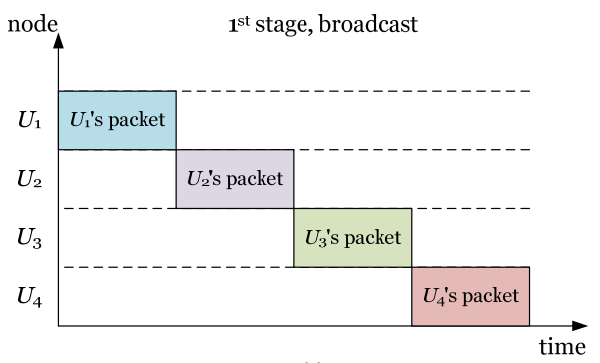

(a)

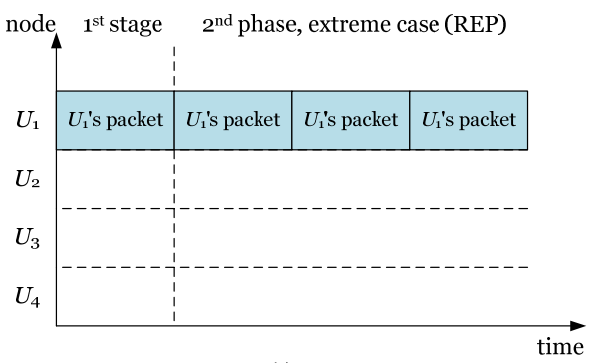

(c)

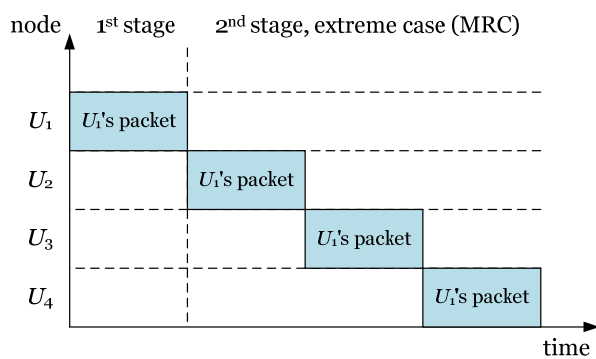

(b)

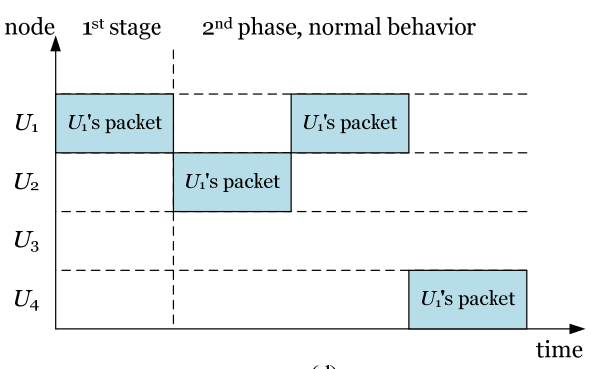

(d)

Fig. 2 Packet scheduling in the two stages of cooperation.

packets which have not been successfully decoded to compensate for the unsuccessfully decoded packets, ensuring the fusion center receives a total of $M$ packets per cooperation frame. The second stage is performed over a total $(M-1)$ time steps. It is assumed that each channel realization remains unchanged during one cooperation frame, but has independent changes from frame to frame, i.e., the network is subject to quasi-static fading. Owing to system simplicity, all average uplink SNRs are considered to be the same. Similarly, the same follows for average internode SNRs. Internode channel reciprocity is assumed, allowing an internode channel realization in the direction from node $U_{i}$ to $U_{j}$ to be the same as in the opposite direction, from $U_{j}$ to $U_{i}$. As a result, the number of re-sent packets per node will be directly related to the number of relayed packets per same node.

Nodes transmit their packets on orthogonal channels allowing the fusion center to detect each node individually, and distinguish from which node a packet is sent, and whether the packet is originating from a node, or just being relayed. Depending on the number of relayed and re-sent data packets, the fusion center applies MRC on the relayed packet copies with the initial packet (which is transmitted in the first phase of cooperation). The remaining (if there exist any) packet copies that are re-sent can be viewed as a single copy with a power gain equal to the number of repetitions [7-9]. These two transmissions are further compared and the fusion center chooses the stronger of the two with a selection combiner (SC). One must take note of the two extreme cases - when there are no retransmissions as in Fig. 2 (b), and when there are no relayed packets as shown in Fig. 2 (c). In the first extreme, the destination applies MRC on the whole $M$ packets copies and automatically chooses that signal (the repeated signal is not present). In the second, however, all packets are coming from one node via the same uplink channel. Instead of choosing between $(M-1)$-fold stronger repeated copy from the second stage and one copy from the first stage, the fusion center will view the whole cooperation frame a single copy with $M$ times the SNR [7]. Fig. 2 (d) shows the case when some of the packets are relayed and some are re-sent.

\section{OUTAGE PROBABILITY ANALYSIS}

Node $U_{i}$ can successfully decode the whole packet if the instantaneous internode SNR, denoted by $\gamma_{i}$, is greater than the optimal threshold SNR $\gamma 6$. The decoding probability can be expressed as

$$
p_{\text {dec }}=\int_{\gamma_{0}}^{\infty} f\left(\gamma_{i}\right) d \gamma_{i}=1-F\left(\gamma_{0}\right),
$$

where $F\left(\gamma_{0}\right)$ is given by (4). When node $U_{i}$ fails to decode $p$ packets, it replaces them with its own packets in resends them to the fusion center. One can treat the $p$ repeated copies as a single copy of the packet with $p$ times the average uplink SNR [9]. The resulting $\operatorname{PDF} f_{\text {rep }}(\gamma \mid p)$ and $\operatorname{CDF} F_{\text {rep }}(\gamma \mid p)$ in this repetition mode will have the same expressions as (3) and (4), respectively, substituting $\bar{\gamma}$ with $p \bar{\gamma}$, and are given as

$$
f_{\text {rep }}(\gamma \mid p)=\frac{2 \Xi_{\text {rep }}^{\frac{k+m}{2}}}{\Gamma(m) \Gamma(k)} \gamma^{\frac{k+m}{2}-1} K_{k-m}\left(2 \sqrt{\Xi_{\text {rep }} \gamma}\right)
$$

and

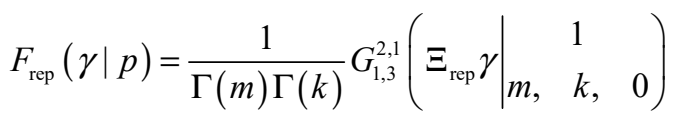

with $\Xi_{\text {rep }}=k m /(p \bar{\gamma})$. These expressions are both conditioned on $p$, which has a binomial distribution

$$
\operatorname{Pr}(p)=\left(\begin{array}{c}
M-1 \\
p
\end{array}\right)\left(1-p_{d e c}\right)^{p} p_{d e c}^{M-p-1} .
$$

At the first level of combining, the fusion center applies $\mathrm{MRC}$ at the relayed packets. It has been shown in [15] that the PDF of the received SNR $\gamma_{\mathrm{mrc}}$ is of an $l$-branch MRC receiver obtained by substituting the fading severity parameter $m$ in (3) with $l m$. In our case, $l=(M-p-1)$, 
and the resulting PDF will be again conditioned on $p$. The CDF of $\gamma_{\mathrm{mrc}}$, will have the same expression as in (4) with argument $\gamma_{0}$, and by substituting $m$ with $(M-p-1) m$ and $\Xi$ with $\Xi_{\mathrm{mrc}}$

$$
\begin{aligned}
F_{\mathrm{mrc}}\left(\gamma_{0} \mid p\right) & =\frac{1}{\Gamma((M-p-1) m) \Gamma(k)} \\
& \times G_{1,3}^{2,1}\left(\left.\Xi_{\mathrm{mrc}} \gamma_{0}\right|_{(M-p-1) m, \quad k,} \quad 0 \begin{array}{c}
1
\end{array}\right),
\end{aligned}
$$

where $\Xi_{\text {mrc }}=k m / \bar{\gamma}$. The fusion center chooses the stronger of the two transmissions when $0 \leq p \leq M-1$, i.e., $\mathrm{SC}$ is performed [20], which for independent branches is a product of the individual outages.

The conditional outage probability can be expressed in terms of the CDFs as a piecewise function, depending on the number of repeated packets as

$$
P_{O}\left(\gamma_{0} \mid p\right)= \begin{cases}F_{\text {mrc }}\left(\gamma_{0} \mid p=0\right), & p=0 \\ F_{\text {mrc }}\left(\gamma_{0} \mid p\right) F_{\text {rep }}\left(\gamma_{0} \mid p\right), & 0 \leq p \leq M-1 \\ F_{\text {rep }}\left(\gamma_{0} \mid p=M-1\right), & p=M-1\end{cases}
$$

Finally, averaging (10) over the number of repeated packets $p$, the expression for outage probability can be expressed as

$$
P_{O}\left(\gamma_{0}\right)=\sum_{p=0}^{M-1} \operatorname{Pr}(p) P_{O}\left(\gamma_{0} \mid p\right)
$$

\section{SPECIAL CASES}

In this Section, the analytical expressions for outage probabilities in "mrc" and "rep" modes in (7) and (9) are reduced for the cases of some commonly used fading models, indicating the generality of the presented outage analysis. The reduced versions of CDF expressions presented in the following subsections, averaged over $p$ account for the outage probability of specific fading and/or shadowing conditions.

\section{A. K fading channel}

By setting $m=1$ in (7) and (9) we obtain the so-called $K$ fading channel, where the multipath component reduces from the Nakagami- $m$ to the Rayleigh distribution, while the shadowing component remains Gamma distributed. The CDF expressions can therefore be expressed as [11]

$$
\begin{aligned}
& F_{\mathrm{mrc}}^{(K \text {-dist })}(\gamma \mid p)=\frac{1}{\Gamma(M-p-1) \Gamma(k)} \\
& \times G_{1,3}^{2,1}\left(\left.\frac{k \gamma_{0}}{\bar{\gamma}}\right|_{M-p-1,} \quad k, \quad 0\right)
\end{aligned}
$$

and

$$
F_{\text {rep }}^{(K-\text { dist })}(\gamma \mid p)=\frac{1}{\Gamma(k)} G_{1,3}^{2,1}\left(\frac{k \gamma_{0}}{p \bar{\gamma}} \mid \begin{array}{ccc}
1, & k, & 0
\end{array}\right) .
$$

\section{B. Nakagami-m channel}

The $K_{G}$ channel reduces to the Nakagami- $m$ channel by letting $k \rightarrow \infty$, diminishing the shadowing component but retaining variable multipath fading severity. By exploiting the permutation symmetry property of the Meijer's $G$ function [19, eq. (07.34.04.0004.01)] and with the help of
[19, eq. (07.34.25.0007.01)] we take the limit of (9) at $k \rightarrow \infty$. After some mathematical manipulations, the CDF for the "mrc" mode over the Nakagami- $m$ fading takes the familiar form [20]

$$
F_{\text {mrc }}^{\text {(naka) }}\left(\gamma_{0} \mid p\right)=1-\frac{\Gamma\left((M-p-1) m, m \gamma_{0} / \bar{\gamma}\right)}{\Gamma((M-p-1) m)},
$$

where $\Gamma(\cdot$,$) is the incomplete Gamma function, defined in$ [18, eq. (8.350.2)]. Moreover, for integer values of the fading severity parameter $m$, the incomplete Gamma functions in (14) reduce to a finite series applying [19, eq. $(06.06 .03 .0009 .01)]$ as

$$
F_{\text {mrc }}^{\text {(naka) }}\left(\gamma_{0} \mid p\right)=1-\exp \left(-\frac{m \gamma_{0}}{\bar{\gamma}}\right)^{(M-p-1) m-1} \sum_{i=0}^{1} \frac{1}{i !}\left(\frac{m \gamma_{0}}{\bar{\gamma}}\right)^{i}
$$

Following the same steps, the CDF expression for "rep" mode over the Nakagami- $m$ channels for non-integer and integer values of $m$ are given respectively as [20]

$$
F_{\text {rep }}^{\text {(naka) }}\left(\gamma_{0} \mid p\right)=1-\frac{\Gamma\left(m, m \gamma_{0} /(p \bar{\gamma})\right)}{\Gamma(m)}
$$

and

$$
F_{\text {rep }}^{\text {(naka) }}\left(\gamma_{0} \mid p\right)=1-\exp \left(-\frac{m \gamma_{0}}{p \bar{\gamma}}\right) \sum_{i=0}^{m-1} \frac{1}{i !}\left(\frac{m \gamma_{0}}{p \bar{\gamma}}\right)^{i}
$$

\section{Rayleigh channel}

Finally, by setting $m=1$ in (15) and (17), the CDF expressions are obtained when the network is subject to the Rayleigh fading. The expressions for "mrc" and "rep" mode are therefore given respectively as [20]

$$
F_{\mathrm{mrc}}^{(\text {ray })}\left(\gamma_{0} \mid p\right)=1-\exp \left(-\frac{\gamma_{0}}{\bar{\gamma}}\right)^{(M-p-1)-1} \sum_{i=0}^{1} \frac{1}{i !}\left(\frac{\gamma_{0}}{\bar{\gamma}}\right)^{i}
$$

and

$$
F_{\text {rep }}^{\text {(ray) }}\left(\gamma_{0} \mid \mathrm{p}\right)=1-\exp \left(-\frac{\gamma_{0}}{p \bar{\gamma}}\right)
$$

\section{NUMERICAL RESUlTS}

The derived outage probability expressions are used to efficiently evaluate numerical results for any practical values of fading severity, shadowing sharpness, average uplink and internode SNR and network cluster size. The outage threshold and the decoding threshold are set to be the same value, which depends on average SNR and packet length $L$. The optimal threshold values for uncoded binary phase shift keyed (BPSK) signals are taken from [11].

All analytical results are confirmed by Monte-Carlo simulations. Simulations were performed using MATLAB software package by generating $10^{7} \mathrm{RVs}$ which are associated with the $K_{G}$ channel model. All commands for generating RVs are built-in into MATLAB. The $K_{G} \mathrm{RV}$ is generated as a product of the square root of two independent Gamma-distributed RVs with shaping parameters $m$ and $k$, respectively. The generated $\mathrm{RV}$ is then compared to the predetermined threshold. Outage probability is finally obtained by counting how many times the generated RV is less than the threshold. In all 
figures, the packet length is set to 512 bits. Choosing smaller or larger packet lengths, the main conclusions remain the same, and only a shift on outage probability values is noticed.

Fig. 3 shows outage probability dependence on shadowing spread for different values of multipath fading severity and average uplink SNR. As expected, by increasing the shadowing spread $\sigma_{S H}$, outage probability grows. This effect is more evident when the uplink channel is more reliable. At high average uplink SNR, in low shadowing, outage probability can be very small, and only in sharp shadowing conditions outage probability can increase by several orders of magnitude. For instance, for $m=2.5$ and when the uplink channel is overall poor, e.g., at $\bar{\gamma}=10 \mathrm{~dB}$, outage probability grows only 10.6 times as shadowing sharpness increases from $3 \mathrm{~dB}$ to $12 \mathrm{~dB}$. However, for reliable uplink channels, at $\bar{\gamma}=30 \mathrm{~dB}$, this increase is even $1.4 \times 10^{5}$ times greater.

The effects of fading severity are shown in Fig. 4. In addition to light and heavy shadowing conditions, two curves corresponding to the case when no shadowing is present (i.e., the Nakagami- $m$ fading) are shown. In heavy shadowing, for $\sigma_{S H}=12 \mathrm{~dB}$, outage probability stays above $10^{-2}$, regardless of the multipath fading severity.

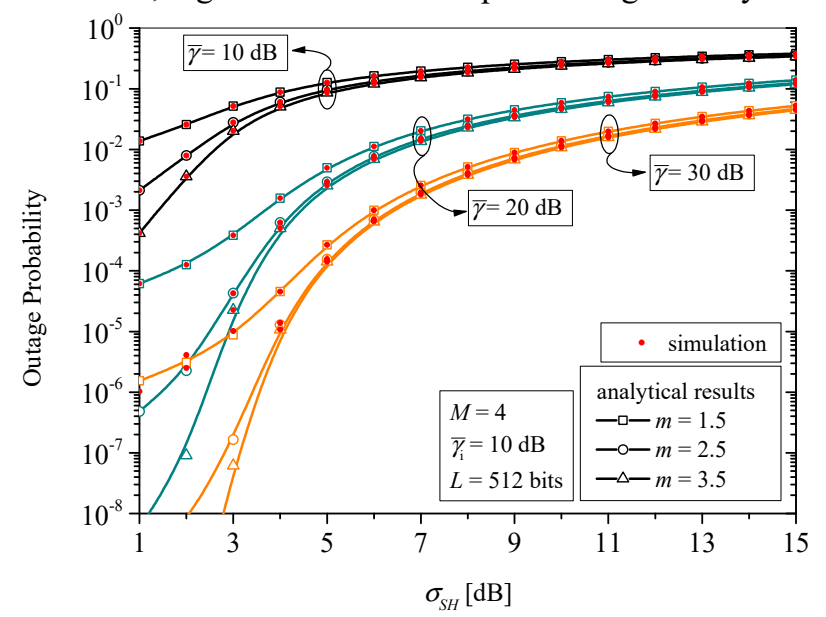

Fig. 3 Outage probability dependence on shadowing spread for different values of fading severity and average uplink SNR.

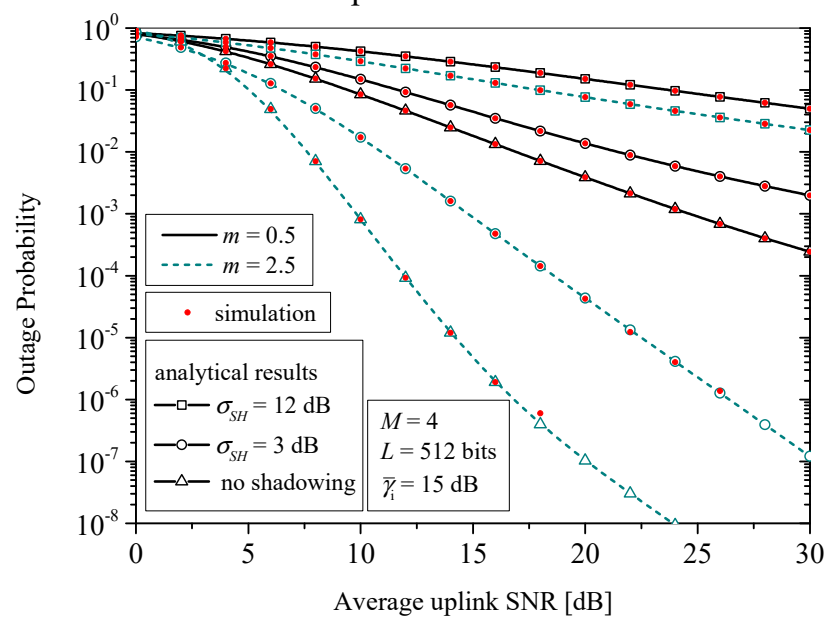

Fig. 4 Outage probability dependence on average uplink SNR for different shadowing sharpness and fading severity.

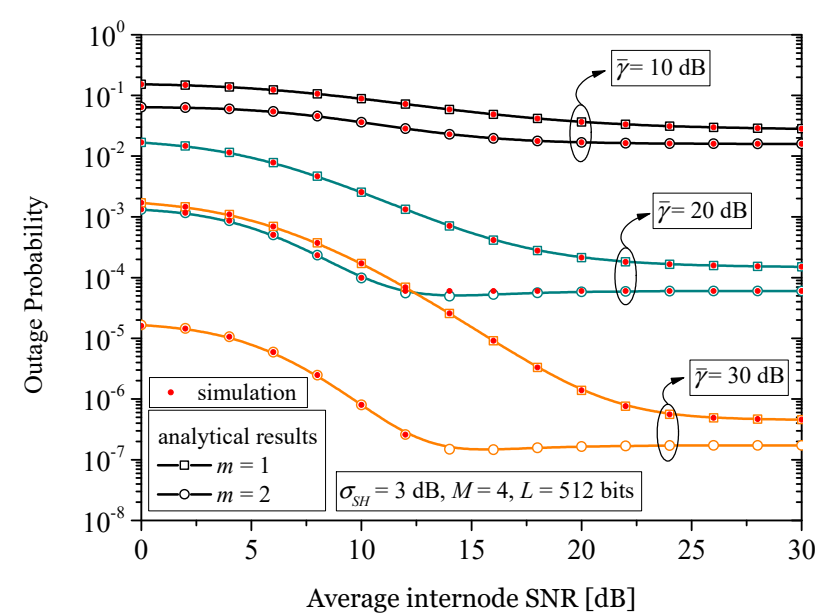

Fig. 5 Outage probability dependence on average internode SNR for different fading severity and average uplink SNR.

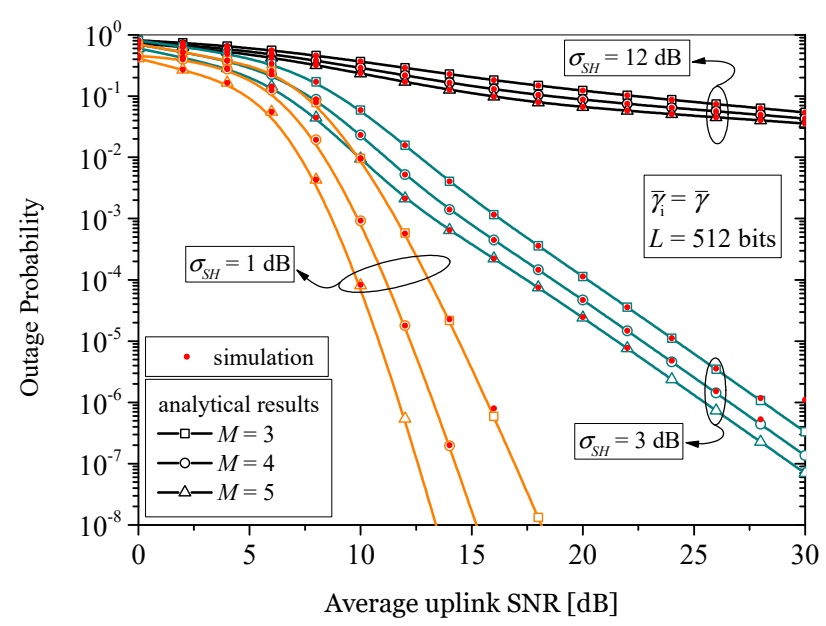

Fig. 6 Outage probability dependence on average uplink SNR for different network size shadowing spread.

Moreover, in severe fading, outage probability is no smaller than $2.4 \times 10^{-4}$, even when no shadowing is present. In favorable fading conditions, i.e., $m=2.5$, outage can be even lower than $10^{-8}$ when no shadowing is present, and around $10^{-7}$ for $\sigma_{S H}=3 \mathrm{~dB}$ and $\bar{\gamma}=30 \mathrm{~dB}$.

Fig. 5 shows outage probability dependence on average internode SNR for different average uplink SNR and fading severity in light shadowing conditions. At low average internode SNR, relays fail to successfully decode the packets and the fusion center chooses the repeated signal more often. Conversely, at high average internode SNR, all nodes have decoded the packets successfully, and the fusion center combines the packets arriving from $M$ independent paths using MRC. For a fixed value of the average uplink SNR, when the internode channels are sufficiently reliable that all packets are successfully decoded, an outage floor appears. Besides the average uplink SNR, the value of this floor is dependent on the fading severity and shadowing sharpness. For instance, at $\bar{\gamma}=30 \mathrm{~dB}$, a floor of $1.7 \times 10^{-7}$ can be noticed at $\bar{\gamma}_{i}=14$ $\mathrm{dB}$ for $m=3$, and a floor of $4.9 \times 10^{-7}$ can be noticed at $\bar{\gamma}_{i}=26 \mathrm{~dB}$ for $m=2$. 
Finally, Fig. 6 shows outage probability dependence on average uplink SNR for different cluster size and shadowing sharpness. Additionally, average internode SNR increases simultaneously with average uplink SNR. As in the previous figures, heavy shadowing will limit outage probability regardless of channel reliability and network size. It is only with the decrease of the effects of shadowing that the gain in adding more nodes to the network cluster can be seen. For instance, to reach an outage of $10^{-5}$, for $M=3$, the average SNR needs to be $24.2 \mathrm{~dB}$, and only $21.4 \mathrm{~dB}$ for $M=5$ at $\sigma_{S H}=3 \mathrm{~dB}$. In even lighter shadowing conditions, i.e., $\sigma_{S H}=1 \mathrm{~dB}$, this outage is reached at an average SNR of $14.4 \mathrm{~dB}$ for $M=3$, and at $10.8 \mathrm{~dB}$ for $M=5$ nodes. Furthermore, the slope of the outage curves is also dominated by the degree of shadowing - as shadowing becomes more light, the diversity gain of the network cluster increases.

\section{CONCLUSION}

In this paper, the outage probability of a decode-andforward cooperative wireless network cluster affected by the Nagakami- $m$ multipath fading and Gamma shadowing has been analyzed. The focus was set on the two-level mechanism at the fusion center, which operates by making a selection between relayed and repeated data packets. Closed-form outage probability expressions have been derived and confirmed by Monte-Carlo simulations. Additionally, these expressions, which have been derived for the generalized- $K$ fading and shadowing channel, have been reduced to $K$, Nakagami- $m$ and Rayleigh fading environments. The impact of uplink and internode channel realizations, shadowing sharpness and multipath fading severity, as well as network size has been examined.

The results have illustrated that the diversity gain of the observed system is affected by the level of shadowing present, and higher diversity gains have been obtained for lighter shadowing. As internode channel conditions improved, outage performance increased until reaching an irreversible outage floor. Moreover, by increasing the number of nodes in the network cluster, only a coding gain has been detected.

The effects of shadowing sharpness on outage probability have shown to be a dominant performance degrading factor, regardless of the multipath fading severity. When no shadowing was present, the existence of a LoS component (i.e., when fading severity decreases) drastically improved performance up to several orders of magnitude when compared to the corresponding cases with shadowing.

\section{REFERENCES}

[1] J. Laneman, D. Tse, and G. W. Wornell, "Cooperative diversity in wireless networks: Efficient protocols and outage behavior," IEEE Trans. Inf. Theory, vol. 50, no. 12, pp. 3062-3080, December 2004.

[2] Y. Zhao, R. Adve, and T. J. Lim, "Outage probability at arbitrary SNR with cooperative diversity," IEEE Commun. Lett., vol. 9, no. 8, pp. 700-702, August 2005.

[3] H. Suraweera, P. Smith, and J. Armstrong, "Outage probability of cooperative relay networks in Nakagami- $m$ fading channels," IEEE Commun. Lett., vol. 10, no. 12, pp. 834-836, December 2006.

[4] J. G. Andrews, S. Buzzi, W. Choi, S. V. Hanly, A. Lozano, A. C. Soong, and J. C. Zhang, "What will 5G be?," IEEE J. Sel. Areas Commun., vol. 32, no. 6, pp. 1065-1082, June 2014.

[5] E. Zimmermann, P. Herhold, and G. Fettweis, "On the performance of cooperative diversity protocols in practical wireless systems," in Proc. 58th IEEE VTC 2003-Fall, Orlando, 2003, pp.2212-2216.

[6] A. Lozano, R. Heath, and J. Andrews, "Fundamental limits of cooperation," IEEE Trans. Inf. Theory, vol. 59, no. 9, pp. 52135226, September 2013.

[7] I. Chatzigeorgiou, W. Guo, I. J. Wassell, and R. A. Carrasco, "Exact and asymptotic outage probability analysis for decode-andforward networks," IEEE Trans. Commun., vol. 59, no. 2, pp. 376 381, February 2011.

[8] I. Chatzigeorgiou, W. Guo, I. J. Wassell, and R. A. Carrasco, "Packet error probability for decode-and-forward cooperative networks of selfish users," in Proc. 10th ISCTA, 2009.

[9] I. Chatzigeorgiou, W. Guo, I. Wassell, and R. Carrasco, "Error probability analysis of unselfish cooperation over quasi-static fading channels," in Proc. 71st IEEE VTC 2010-Spring, Taipei, 2010, pp.1-5.

[10] N. Zdravković, A. Cvetkovic, K. Kansanen, and G. T. Djordjevic, "Outage performance of low-latency decode-and-forward cooperative wireless networks," EURASIP J. Wirel. Commun. Netw., vol. 2016, no. 1, pp. 1-10, April 2016.

[11] N. Zdravković, "Outage analysis in clustered cooperative networks over $K$ fading channels," in Proc. 23rd TELFOR, Belgrade, 2015, pp.95-98.

[12] A. Dziri, M. Terre, N. Nasser, "Performance analysis of decode and forward cooperative relaying over the generalized-K channel," Wireless Engineering and Technology, vol. 4, no. 2, pp.92-100, April 2013.

[13] E. Tanghe, W. Joseph, L. Verloock, L. Martens, H. Capoen, K. Van Herwegen, and W. Vantomme, "The industrial indoor channel: large-scale and temporal fading at 900,2400, and $5200 \mathrm{Mhz}$," IEEE Trans. Wireless Commun., vol. 7, no. 7, pp. 2740-2751, July 2008.

[14] P. Shankar, "Error rates in generalized shadowed fading channels," Wireless Pers. Commun., vol. 28, no. 3, pp. 233-238, February 2004.

[15] P. Shankar, "Outage probabilities of a MIMO scheme in shadowed fading channels with micro- and macrodiversity reception," IEEE Trans. Wireless Commun., vol. 7, no. 6, pp. 2015-2019, June 2008.

[16] I. Kostic, "Analytical approach to performance analysis for channel subject to shadowing and fading," IEE Proc. Commun., vol. 152, no. 6, pp. 821-827, December 2005.

[17] P. Bithas, N. Sagias, P. Mathiopoulos, G. Karagiannidis, and A. Rontogiannis, "On the performance analysis of digital communications over generalized- $K$ fading channels," IEEE Commun. Lett., vol. 10, no. 5, pp. 353-355, May 2006.

[18] I. Gradshteyn and I. Ryzhik, Table of Integrals, Series, and Products, 5th edition. Academic Press, 1994.

[19] The wolfarm functions site. [Online]. Available: http://functions.wolfram.com/

[20] M. K. Simon and M.-S. Alouini, Digital communication over fading channels. New York: John Wiley \& Sons, 2005.

[21] N. Zdravković, "Outage analysis in clustered cooperative networks over K fading channels," Telecommunications Forum Telfor (TELFOR), 2015 23rd, Belgrade, 2015, pp. 95-98. 